\title{
A PROLONGATION/RESTRICTION OPERATOR FOR WHITNEY ELEMENTS ON SIMPLICIAL MESHES*
}

\author{
ALAIN BOSSAVIT ${ }^{\dagger}$ AND FRANCESCA RAPETTI ${ }^{\ddagger}$
}

\begin{abstract}
The paper is mainly focused on the construction of two transfer operators between nested grids in the case of Whitney finite elements (node-, edge-, face-, or volume-based). These transfer operators, instances of what is called "chain map" in homology, have duals acting on cochains, that is to say, arrays of degrees of freedom in the context of the finite-element discretization of variational problems. We show how these duals can act as restriction/prolongation operators in a multigrid approach to such problems, especially those involving vector fields (e.g., electromagnetism). The duality between the operation of mesh refinement of a simplicial complex and that of restriction/prolongation of degrees of freedom from one mesh to a nested one is thus analyzed and explained. We use the language of $p$-forms, with frequent explanatory references to the more traditional vector-fields formalism.
\end{abstract}

Key words. mesh refinement, $p$-chains, $p$-forms, Whitney elements, multigrid

AMS subject classification. 65 N30

DOI. $10.1137 / 040604923$

1. Introduction. In the approximation of a given differential problem by a finite element method, solving the final algebraic linear system is a delicate step. It is wellknown that the associated matrix is sparse and can be of large size so that iterative solvers are preferable to direct ones. However, the convergence of iterative solvers strongly depends on the matrix condition number and slows down when the latter is large. Moreover, classical iterative methods fail to be effective whenever the spectral radius of the iteration matrix is close to one. A Fourier analysis shows that the reduction in the error depends on the spatial frequency. Errors with high frequency are rapidly damped whereas low frequency errors are slowly reduced and hold back convergence.

The multigrid algorithm [16] is an iterative technique well-adapted to solving linear systems arising from a finite element discretization of differential equations over a given grid. The basic idea of the method is to change the grid in such a way that low frequency (smooth) errors on a grid with elements of maximal diameter $h$ can be singled out and cut down on a coarser grid, while high frequency errors that are not visible on the coarse grid with elements of maximal diameter $H>h$, for example, can be resolved on the fine grid. The exchange of information between the two meshes is done by means of two linear operators, one behaving as a prolongation and the other as a restriction. These operators are well known for nodal finite elements on nested or nonnested grids [19] but have still to be fully understood for edge or face finite elements.

It must be remarked that recovering the coarse grid from the fine one can be a very demanding operation. Therefore, in this paper we will address this problem the other

*Received by the editors March 9, 2004; accepted for publication (in revised form) November 1, 2004; published electronically December 15, 2005.

http://www.siam.org/journals/sinum/43-5/60492.html

†Laboratoire de Génie Electrique de Paris, UMR 8507 CNRS, Plateau de Moulon, Gif-sur-Yvette, Paris 91192, France (bossavit@lgep.supelec.fr).

${ }^{\ddagger}$ Laboratoire de Mathématiques, UMR 6621 CNRS and Université de Nice et Sophia-Antipolis, Parc Valrose, 06108 Nice cedex 02, France (frapetti@math.unice.fr). 
way around; i.e., we suppose that we have a coarse grid and we refine it repeatedly by means of a fixed procedure. This way, by using a suitable system of labels for the mesh nodes, we can know at which refinement level we are. Any other situation is not considered here since we wish to focus on the transfer of degrees of freedom from one mesh to the other, rather than on the coarsening process itself. However, the proposed analysis does not depend on the refinement or coarsening process. In short, we focus on a specific criterion to present the theory, but the theory is independent from the chosen criterion.

The paper is organized as follows. In section 2, suitable algebraic tools are introduced to lead the reader into the "world" of Whitney elements on simplicial meshes, including an appropriate formulation of the Stokes theorem. In section 3, we consider the problem of subdividing a simplicial mesh. The core of the paper is section 4, where we construct the information exchange operators between two "nested" meshes (by which we mean, two meshes $m$ and $\tilde{m}$, the latter a conforming refinement of the former). Notions thus developed are applied in section 5, where we define the two transfer operators for Whitney elements on two nested simplicial meshes. The multigrid algorithm then comes as a straightforward application of these notions. Analyzing its performances is a difficult and technical issue, which we do not address. (Relevant references are given in section 5.)

2. Algebraic tools. In this section, we recall some basic notions in algebraic topology (see, e.g., $[1,17])$ and explain our notation. We restrict ourselves to a threedimensional domain $\Omega$ (but the same notions can be defined in any dimension). For all integrals, we omit specifying the integration variable when this can be done without ambiguity. We shall denote by $\int_{\gamma} \mathbf{u} \cdot \mathbf{t}_{\gamma}$ and $\int_{\sigma} \mathbf{u} \cdot \mathbf{n}_{\sigma}$, respectively, the circulation and the flux of a vector field $\mathbf{u}$, where $\mathbf{t}_{\gamma}$ is the unit tangent to the smooth curve $\gamma$ and $\mathbf{n}_{\sigma}$ the outward unit normal to the surface $\sigma$. Moreover, we shall emphasize the maps $\gamma \rightarrow \int_{\gamma} \mathbf{u} \cdot \mathbf{t}_{\gamma}$ and $\sigma \rightarrow \int_{\sigma} \mathbf{u} \cdot \mathbf{n}_{\sigma}$, that is to say, the differential forms of degree 1 and 2 , respectively, which one can associate with a given vector field $\mathbf{u}$, and we occasionally use notations specific to exterior calculus, such as the exterior derivative d, as used in the Stokes theorem.

2.1. Triangulations and Whitney finite elements. Given a domain $\Omega \subset \mathbb{R}^{3}$ with boundary $\Gamma$, a simplicial mesh $m$ in $\Omega$ is a tessellation of $\bar{\Omega}$ by tetrahedra, under the condition that any two of them may intersect along a common face, edge, or node, but in no other way. We denote by $\mathcal{N}_{m}, \mathcal{E}_{m}, \mathcal{F}_{m}, \mathcal{T}_{m}$ (nodes, edges, faces, and tetrahedra, respectively) the sets of simplices of dimension 0 to 3 thus obtained (see Figure 1 for an example), each with its own orientation (more on this will follow), and by $N_{m}, E_{m}, F_{m}, T_{m}$ their cardinalities. Alternatively, we may use $\mathcal{S}_{m}^{p}$ to denote the set of $p$-dimensional simplices in $m$ and $\# \mathcal{S}_{m}^{p}$ for its cardinality. The importance of simplicial meshes lies in the fact that any triangulated domain is homeomorphic to one in which the triangles are flat and the edges straight. Note that the triangulation for $\Omega$ is not unique, but topological properties of a triangulated domain do not depend on the triangulation used to investigate them. (For such "homological" computations, using a definite triangulation but yielding mesh-independent results, which we believe are relevant to engineering practice, see $[12,15]$.)

For what follows, we need to underline some combinatorial properties of the simplicial mesh. Besides the list of nodes and their positions, the mesh data structure also contains incidence matrices, saying which node lies at an end of which edge, which edge bounds which face, etc. [4]. This encodes the orientation of each simplex, as we now explain. In short, an oriented edge is not only a two-node subset of $\mathcal{N}_{m}$, 

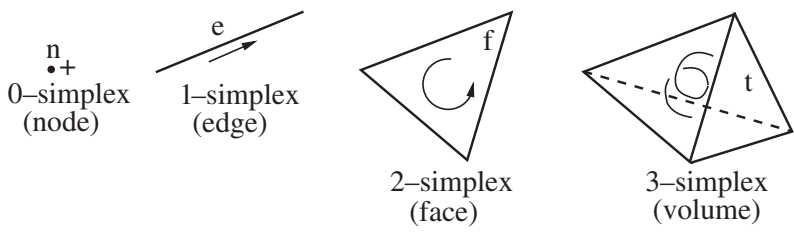

FIG. 1. Examples of oriented $p$-simplex, $p=0, \ldots, 3$.

but an ordered such set, where the order implies an orientation. Let $e=\{\ell, n\}$ be an edge of the mesh oriented from the node $\ell$ to $n$. We can define the incidence numbers $G_{e, n}=1, G_{e, \ell}=-1$, and $G_{e, k}=0$ for all nodes $k$ other than $\ell$ and $n$. These numbers form a $\left(E_{m} \times N_{m}\right)$-matrix $G$, which describes how edges connect to nodes. A face $f=\{\ell, n, k\}$ has three vertices which are the nodes $\ell, n, k$. Note that $\{n, k, l\}$ and $\{k, l, n\}$ denote the same face $f$, whereas $\{n, l, k\}$ denotes an oppositely oriented face, which is not supposed to belong to $\mathcal{F}_{m}$ if $f$ does. An orientation of $f$ induces an orientation of its boundary. So, with respect to the orientation of the face $f$, the one of the edge $\{l, n\}$ is positive and that of $\{k, n\}$ is negative. So we can define the incidence number $R_{f, e}=1$ (resp., -1) if the orientation of $e$ matches (resp., does not match) the one on the boundary of $f$ and $R_{f, e}=0$ if $e$ is not an edge of $f$. These numbers form a $\left(F_{m} \times E_{m}\right)$-matrix $R$. Finally, let us consider the tetrahedron $t=\{k, l, m, n\}$, positively oriented if the three vectors $\{k, l\},\{k, m\}$, and $\{k, n\}$ define a positive frame $\left(t^{\prime}=\{l, m, n, k\}\right.$ has a negative orientation and does not belong to $\mathcal{T}_{m}$ if $t$ does). A $\left(V_{m} \times F_{m}\right)$-matrix $D$ can be defined by setting $D_{t, f}= \pm 1$ if face $f$ bounds the tetrahedron $t$, with the sign depending on whether the orientation of $f$ and of the boundary of $t$ match or not, and $D_{t, f}=0$ in case $f$ does not bound $t$. (For consistency, we may attribute an orientation to nodes as well-a sign, \pm 1 . Implicitly, we have been orienting all nodes the same way $(+1)$ up to now. Note that a sign $(-1)$ to node $n$ changes the sign of all entries of column $n$ in the above $G$.) It can easily be proved that $R G=0$ and $D R=0$ [4].

We now define the Whitney finite elements we use $[4,9,10,13]$ : They are scalar functions or vector fields associated to all the simplices of the mesh $m$. Given the node $n$, the edge $e=\{\ell, m\}$, the face $f=\{\ell, m, k\}$, and the tetrahedron $t=\{i, j, k, \ell\}$, we define the following scalar or vector functions $\left(\lambda_{n}\right.$ is the barycentric coordinate associated to node $n$ ):

$$
\begin{aligned}
w^{n}= & \lambda_{n}, \\
w^{e}= & \lambda_{\ell} \operatorname{grad} \lambda_{m}-\lambda_{m} \operatorname{grad} \lambda_{\ell}, \\
w^{f}= & 2\left(\lambda_{\ell} \operatorname{grad} \lambda_{m} \times \operatorname{grad} \lambda_{k}+\lambda_{m} \operatorname{grad} \lambda_{k} \times \operatorname{grad} \lambda_{\ell}+\lambda_{k} \operatorname{grad} \lambda_{\ell} \times \operatorname{grad} \lambda_{m}\right), \\
w^{t}= & 6\left(\lambda_{i} \operatorname{grad} \lambda_{j} \times \operatorname{grad} \lambda_{k} \cdot \operatorname{grad} \lambda_{\ell}+\lambda_{j} \operatorname{grad} \lambda_{k} \times \operatorname{grad} \lambda_{\ell} \cdot \operatorname{grad} \lambda_{i}\right. \\
& \left.+\lambda_{k} \operatorname{grad} \lambda_{\ell} \times \operatorname{grad} \lambda_{i} \cdot \operatorname{grad} \lambda_{j}+\lambda_{\ell} \operatorname{grad} \lambda_{i} \times \operatorname{grad} \lambda_{j} \cdot \operatorname{grad} \lambda_{k}\right)
\end{aligned}
$$

$\left(w^{t}\right.$ is just the constant $\left.1 / \operatorname{vol}(t)\right)$. We define $W_{m}^{p}=\operatorname{span}\left\{w^{s}: s \in \mathcal{S}_{m}^{p}\right\}, p=0,1,2,3$ (the simplicial dimension, e.g., $p=0$ for nodes). It can be verified that the value (resp., circulation, flux, integral) of $w^{n}$ (resp., $w^{e}, w^{f}, w^{t}$ ) on its supporting simplex is 1 , and 0 on other simplices of matching dimension, a fact we shall be able to state more compactly in a moment.

Given two adjacent tetrahedra $t$ and $t^{\prime}$ sharing a face $f$, the function $w^{n}$ and both the tangential component of $w^{e}$ and the normal component of $w^{f}$ are continuous 
across $f$, whereas the function $w^{t}$ is not. Thanks to these continuity properties, $W_{m}^{0} \subset H^{1}(\Omega), W_{m}^{1} \subset H(\operatorname{curl}, \Omega), W_{m}^{2} \subset H(\operatorname{div}, \Omega), W_{m}^{3} \subset L^{2}(\Omega)$. The spaces $W_{m}^{p}, p=0,1,2,3$, have finite dimension given by $N_{m}, E_{m}, F_{m}, T_{m}$, respectively, and they play the role of Galerkin approximation spaces for the functional spaces just mentioned. Therefore, a scalar field $k \in H^{1}(\Omega)$ can be represented in $W_{m}^{0}$ by the approximation $\sum_{n \in \mathcal{N}_{m}} k_{n} w^{n}$ where $\left\{k_{n}: n \in \mathcal{N}_{m}\right\}$ are the values of $k$ at the mesh nodes (i.e., the degrees of freedom of $k$ on the mesh $m$ ). Similarly, a vector field $\mathbf{v} \in H(\mathbf{c u r l}, \Omega)$ can be represented in $W_{m}^{1}$ by $\sum_{e \in \mathcal{E}_{m}} v_{e} w^{e}$, where $\left\{v_{e}: e \in \mathcal{E}_{m}\right\}$ are the circulations of $\mathbf{v}$ along the mesh edges. A vector field $\mathbf{v} \in H(\operatorname{div}, \Omega)$ can be represented in $W_{m}^{2}$ by $\sum_{f \in \mathcal{F}_{m}} v_{f} w^{f}$, where $\left\{v_{f}: f \in \mathcal{F}_{m}\right\}$ are the fluxes of $\mathbf{v}$ across the mesh faces. Finally, a scalar field $k \in L^{2}(\Omega)$ can be represented in $W_{m}^{3}$ by $\sum_{t \in \mathcal{T}_{m}} k_{t} w^{t}$, where $\left\{k_{t}: t \in \mathcal{T}_{m}\right\}$ are the integrals of $k$ on the mesh tetrahedra.

Properties discussed so far concern the spaces $W_{m}^{p}$ taken one by one. Properties of the structure made of the spaces $W_{m}^{p}$ when taken together should also be mentioned. We know that the following inclusions hold:

$$
\operatorname{grad} W_{m}^{0} \subset W_{m}^{1}, \quad \operatorname{curl} W_{m}^{1} \subset W_{m}^{2}, \quad \operatorname{div} W_{m}^{2} \subset W_{m}^{3} .
$$

It is natural to ask whether the sequence

$$
\{0\} \longrightarrow W_{m}^{0} \stackrel{\text { grad }}{\longrightarrow} W_{m}^{1} \stackrel{\text { curl }}{\longrightarrow} W_{m}^{2} \stackrel{\text { div }}{\longrightarrow} W_{m}^{3} \longrightarrow\{0\}
$$

is exact at levels 1 and 2, i.e., when it happens that

$$
\operatorname{ker}\left(\operatorname{curl} ; W_{m}^{1}\right)=\operatorname{grad} W_{m}^{0}, \quad \operatorname{ker}\left(\operatorname{div} ; W_{m}^{2}\right)=\operatorname{curl} W_{m}^{1},
$$

where $\operatorname{ker}\left(\operatorname{curl} ; W_{m}^{1}\right):=W_{m}^{1} \cap \operatorname{ker}(\mathbf{c u r l})$ and $\operatorname{ker}\left(\operatorname{div} ; W_{m}^{2}\right):=W_{m}^{2} \cap \operatorname{ker}(\operatorname{div})$. (At level 0 , the gradient operator is not injective. At level 3 , the divergence operator is surjective.) The Poincare lemma states that, when the domain $\Omega$ is contractible, the image fills the kernel in both cases. This may fail to happen: With $\Omega$ a solid torus, for example, $\operatorname{grad}\left(W_{m}^{0}\right)$ is a proper subset of $\operatorname{ker}\left(\operatorname{curl} ; W_{m}^{1}\right)$. If so, it tells us something on the topology of $\Omega$, namely the presence of $b_{1}$ "loops," where $b_{1}=$ $\operatorname{dim}\left[\operatorname{ker}\left(\operatorname{curl} ; W_{m}^{1}\right) / \operatorname{grad}\left(W_{m}^{0}\right)\right]$ is the Betti number of dimension 1 of the domain. Solenoidal fields that are not curls indicate the presence of $b_{2}$ "holes," where $b_{2}=$ $\operatorname{dim}\left[\operatorname{ker}\left(\operatorname{div} ; W_{m}^{2}\right) / \operatorname{curl}\left(W_{m}^{1}\right)\right]$ is the Betti number of dimension 2 of the domain. (One may add that $b_{0}=\operatorname{dim}\left[\operatorname{ker}\left(\operatorname{grad} ; W_{m}^{0}\right)\right]$ is the number of connected components, here assumed to be 1, of $\Omega$.) These are global topological properties of the meshed domain: They depend on $\Omega$, but not on which mesh is used to compute them. The sequences are thus an algebraic tool by which the topology of $\Omega$ can be explored (which was the point of inventing Whitney forms [18]).

2.2. Chains and homology groups. We now introduce chains over the mesh $m$. A $p$-chain $c$ is an assignment to each $p$-simplex $s$ of a rational integer $\alpha_{s}$. This can be denoted by $c=\sum_{s \in \mathcal{S}_{m}^{p}} \alpha_{s} s$. Let $C_{p}(m)$ be the set of all $p$-chains. This set has a structure of Abelian group with respect to the addition of $p$-chains: Two $p$-chains are added by adding the corresponding coefficients.

If $s$ is an oriented simplex, the elementary chain corresponding to $s$ is the assignment $\alpha_{s}=1$ and $\alpha_{s}^{\prime}=0$ for all $s^{\prime} \neq s$. In what follows, we will use the same symbol $s$ (or $n, e$, etc., depending) to denote the oriented simplex and the associated elementary chain. Note how this is consistent with the above expansion of $c$ as a formal weighted sum of simplices. Which meaning is implied will hopefully be clear from the context. 
The boundary of an oriented $p$-simplex of $m$ is a $(p-1)$-chain determined by the sum of its $(p-1)$-dimensional faces, each taken with the orientation induced from that of the whole simplex. So, the boundary $\partial s$ of a single simplex $s$ is

$$
\partial e=\sum_{n \in \mathcal{N}_{m}} G_{e, n} n, \quad \partial f=\sum_{e \in \mathcal{E}_{m}} R_{f, e} e, \quad \partial t=\sum_{f \in \mathcal{F}_{m}} D_{t, f} f .
$$

By linearity, the boundary operator $\partial$ defines a group homomorphism $C_{p}(m) \rightarrow$ $C_{p-1}(m)$ as follows:

$$
\partial c=\partial\left(\sum_{s \in \mathcal{S}_{m}^{p}} \alpha_{s} s\right)=\sum_{s \in \mathcal{S}_{m}^{p}} \alpha_{s} \partial s .
$$

Note that $\partial$ is represented by a matrix, which is $G^{t}, R^{t}$, or $D^{t}$, depending on the dimension $p>0$. We remark that $\partial \circ \partial=0$, i.e., the boundary of a boundary is the null chain. When $p=0$, we define the boundary of a single vertex to be zero and $C_{-1}(m)=\{0\}$.

The kernel of $\partial: C_{p}(m) \rightarrow C_{p-1}(m)$ is denoted by $Z_{p}(m)$ and is called the group of $p$-cycles of $m$. The image of $\partial: C_{p+1}(m) \rightarrow C_{q}(m)$ is denoted by $B_{q}(m)$ and is called the group of $p$-boundaries of $m$. The property $\partial \circ \partial=0$ implies that $B_{p}(m)$ is a subgroup of $Z_{p}(m)$. The quotient $H_{p}(m)=Z_{p}(m) / B_{p}(m)$ is the homology group of order $p$ of the mesh $m$ and the Betti number $b_{p}$ is equal to the rank of $H_{p}(m)$. Not all cycles bound, as a rule (think again of the solid torus, for $p=1$ ), so $b_{p}$ need not be zero.

By linearity, integration over simplices extends to chains as follows (let's deal with 2-chains for definiteness). If $c=\Sigma_{f \in \mathcal{F}_{m}} c_{f} f$, the integral of a vector field $w$ over $c$ is, by definition (and with some notational abuse for which we shall be rewarded later),

$$
\int_{c} w=\sum_{f \in \mathcal{F}_{m}} c_{f} \int_{f} w \cdot \mathbf{n}_{f}
$$

Substituting the Whitney form $w^{f}$ for $w$ there, one sees that $\int_{c} w^{f}$ is just $c_{f}$. A similar definition can be stated for node-based, edge-based, or volume-based chains. So we now have $\int_{s^{\prime}} w^{s}=1$ if $s^{\prime}=s$ and 0 if $s^{\prime} \neq s$ for all $p$-simplices $s^{\prime}$ and Whitney elements $w^{s}$ - the promised compact expression of their main property.

Remark 2.1. We note that (1) amounts to considering the vector field $w$ as a differential form, as defined at the beginning of section 1 . The functions and vector fields $w^{n}, w^{e}, w^{f}, w^{t}$ of section 2.1 are thus differential forms, known as Whitney forms in the mathematical literature [18].

2.3. Cochains and cohomology groups. In this section, we introduce the dual concept of $p$-cochain. A p-cochain is a linear functional on the vector space of $p$-chains. For instance, given an array $\mathbf{b}=\left\{b^{s}: s \in \mathcal{S}_{m}^{p}\right\}$ of real numbers, we can define the $p$-cochain $c \rightarrow \sum_{s \in \mathcal{S}_{m}^{p}} b^{s} c_{s}$ acting on $p$-chains $c$ with coefficients $c_{s}$. Also, as in (1), given a differential form $w$, the mapping $c \rightarrow \int_{c} w$ defines a $p$-cochain. More generally, the $p$-cochain coefficients are obtained by integrating the differential form $w$ on the elements of the $p$-chain $c$; i.e., the map $c \rightarrow \sum_{s \in \mathcal{S}_{m}^{p}} c_{s} \int_{s} w$ is a cochain. We shall denote the latter value as $\langle w ; c\rangle$.

Once a metric is introduced on the ambient affine space, differential forms are in correspondence with scalar and vector fields (called "proxy fields" - metric dependent, of course). The coefficients of $p$-cochains become the degrees of freedom of scalar 
and vector fields (and this is exactly what occurs with Whitney finite elements in section 2.1). Let $W^{p}(m)$ denote the set of $p$-cochains (or $p$-forms) defined on $\Omega$ when triangulated by $m$. Then, $C_{p}(m)$ and $W^{p}(m)$ are in duality via the bilinear continuous $\operatorname{map}\langle\cdot ; \cdot\rangle: W^{p}(m) \times C_{p}(m) \rightarrow \mathbb{R}$ defined as $\langle w ; c\rangle=\int_{c} w$ where the integral must be interpreted as in (1) in the example case $p=2$. A duality product should be nondegenerate, i.e., $\langle w ; c\rangle=0$ for all $c$ implies $w=0$, and $\langle w ; c\rangle=0$ for all $w$ implies $c=0$. The former property holds true by definition, and the latter is satisfied because, if $c \neq 0$, one can construct an ad-hoc smooth vector field or function with nonzero integral and hence a nonzero form $w$ such that $\langle w ; c\rangle \neq 0$.

For $p>0$, the exterior derivative of the $(p-1)$-form $w$ is the $p$-form $\mathrm{d} w$. The integral $\int_{c} w$ is treated in two ways: If $c=\partial \tau$ and $w$ is smooth, one may go forward and integrate $d w$ over $\tau$. Alternatively, if the form $w=d v$, one may go backward and integrate $v$ over $\partial c$. In particular, we have $\int_{\partial c} w=\int_{c} \mathrm{~d} w$, which is the common form of Stokes' theorem [7], or equivalently,

$$
\langle w ; \partial c\rangle=\langle\mathrm{d} w ; c\rangle \quad \forall c \in C_{p} \quad \text { and } \quad \forall w \in W^{p-1} .
$$

Equation (2) reveals that $\mathrm{d}$ is the dual of $\partial$ (in the sense of Yosida [20, p. 194]). As a corollary of the boundary operator property $\partial \circ \partial=0$, we have that $\mathrm{d} \circ \mathrm{d}=0$. A form $w$ is closed if $\mathrm{d} w=0$, exact if $w=\mathrm{d} v$ for some $v$ (in the first case we have a cocycle and in the second case a coboundary). Denoting by $\mathcal{Z}^{p}(m)$ the vector space of all closed $p$-forms and by $\mathcal{B}^{p}(m)$ the subspace made of all exact $p$-forms, the property $\mathrm{d} \circ \mathrm{d}=0$ implies that $\mathcal{B}^{p}(m) \subset \mathcal{Z}^{p}(m)$; i.e., the integral of a cocycle over a boundary vanishes. In domains $\Omega$ that are topologically trivial, all closed $p$-forms are exact (this is the Poincaré lemma). But closed forms need not be exact in general manifolds: This is the dual aspect of the above "not all cycles bound" (section 2.2). The quotient space $\mathcal{H}^{p}(m)=\mathcal{Z}^{p}(m) / \mathcal{B}^{p}(m)$ is (considered as an additive group) the De Rham's pth cohomology group of $\Omega$ or equivalently of $m$.

3. Refinement of a triangulation and simplicial maps. A mesh refinement is a procedure to subdivide each simplex of a given mesh (referred to as the "coarse" one) $m$ into a finite number of smaller ones, whose assembly is still a proper mesh (the "fine" one). We consider here conforming refinements, i.e., such that the set $\tilde{m}$ of all simplices of the fine mesh, is itself a cellular complex (no hanging nodes). Moreover, we are interested in subdividing a simplicial mesh in a way that will not deteriorate the aspect ratio of the new smaller and smaller tetrahedra that appear during the division process. In this framework, we speak of uniform refinement procedure if there is a finite catalog of "model cells" such that any cell in any $\tilde{m}$ is similar to one of them, for all meshes $\tilde{m}$ in the family $\mathcal{M}$ of meshes potentially created in the process of iterated refinement.

The barycentric (or regular) refinement is an example of conforming refinement procedure where the small cells are more and more stretched (see Figure 2 for a face) and hence not uniform in that sense. In three dimensions, each tetrahedron $T$ is divided into 24 tetrahedra, and we can understand that after the first step of refinement, the new tetrahedra are more stretched toward the barycenter $o$ : When their aspect ratio becomes too small, the classical a priori error estimates for finite elements do not apply and convergence is not warranted. 

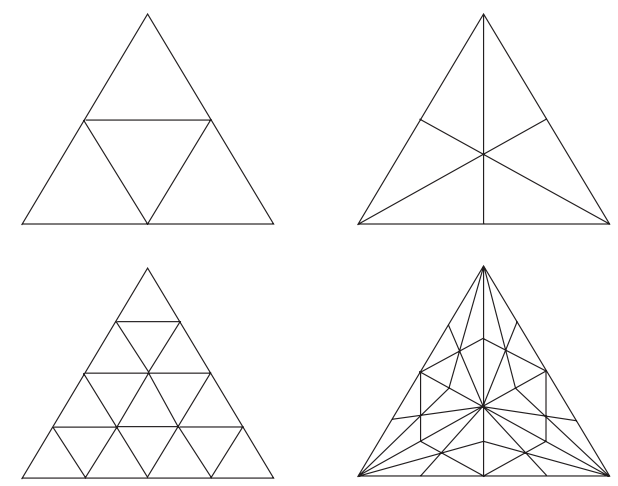

FIG. 2. Two-level refinement of normal (left) and barycentric (right) type. A face is divided, respectively, into four faces (left) and six faces (right) at each refinement level.

The normal refinement ${ }^{1}$ presented in Figure 2 for a face, and in Figure 5 for a tetrahedron, is an example of conforming refinement procedure that enjoys uniformity. In three dimensions, let us consider a tetrahedron $T$ built on four nodes $k, l, m, n$. Call $o$ the center, $l m, l n$, etc., the midpoints. The big tetrahedron $T=\{k, l, m, n\}$ subdivides into four midsize ones, such as $\{k n, l n, m n, n\}$, and a midsize core octahedron (Figure 3), itself a half-size reduction of a big one $O$ circumscribed to $T$. In turn, the core divides into six small octahedra and eight small tetrahedra, all similar to $O$ and $T$, respectively, with a reduction factor of 4 (Figure 4). Hence there are two basic shapes, that of $T$ and that of $O$, which are found again and again.
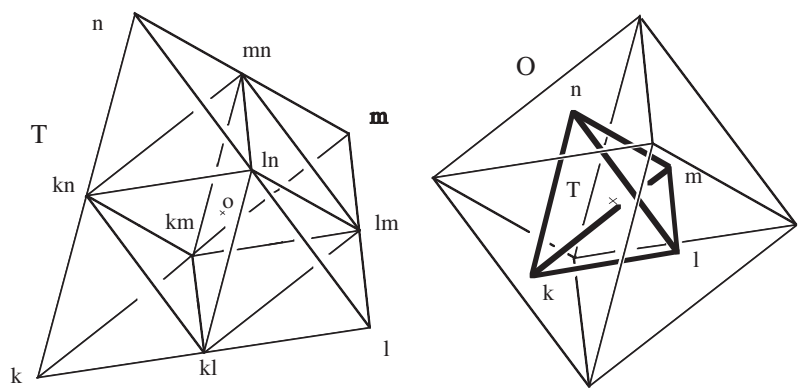

FIG. 3. Cutting $T$ into four midsize tetrahedra plus a core octahedron, similar to the circumscribed one, $O$. Note that the common center o of $T$ and $O$ is four times closer to face $\{k, l, m\}$ than node $n$ was. Faces of $O$ are similar to those of $T$, twice as big.

All that is left to do, in order to get a series of nested simplicial meshes, is to cut the octahedra into tetrahedra, either eight (Figure 5) or just four. The latter solution simply consists of adding an edge joining two opposite nodes of the octahedron. As there are three nonequivalent ways to do that, one must be careful to draw all these

\footnotetext{
${ }^{1}$ Whitney defines in [18, pp. 358-360] a standard subdivision that guarantees uniformity but does not treat nodes symmetrically, the way ours does, hence the introduction of the adjective normal for definiteness. Normal subdivision can be done in dimensions $d>3$, where it also involves, as can be inferred from Figure 3, convex hulls of barycenters of $p$-faces of the reference $d$-simplex. Denoting such convex polytopes by $T_{p}$ (the reference simplex thus being $T_{0}$, and the $O$ of Figure 5 a $T_{1}$ ), it can be shown that each $T_{p}$ can be dissected into a finite number (bounded by a function of $d$ only) of polytopes similar to one of the $T_{q}, 1 \leq q \leq d$; hence there is uniformity.
} 


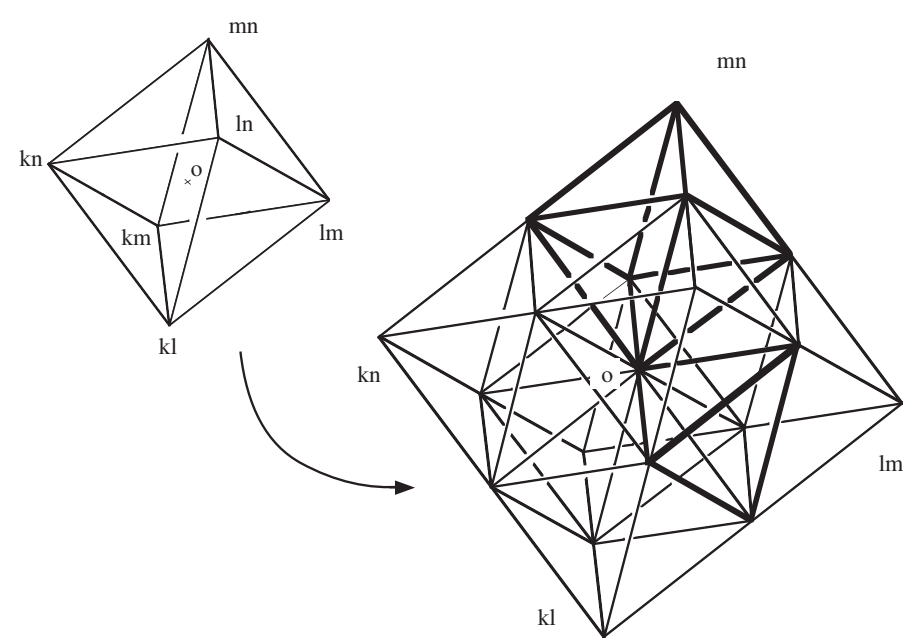

FIG. 4. Cutting the octahedral core $O$ into six small octahedra (one per node of the core, or edge of $T$ ) plus eight small tetrahedra (one per face of the core), all similar to $T$ and $O$, respectively, and four times smaller.

diagonals parallel to a same direction if one wants to minimize the number of different shapes of tetrahedra. Cutting in eight is a more symmetrical procedure. With both methods, the number of shapes is kept down to five. (That is the generic number, of course lower if $T$ had some symmetry to start with.)

In any event, it is only at the latest stage of the subdivision that the final cut of octahedra should be involved. Conceptually, we have two cell shapes, $T$ and $O$. Each $T$-cell breaks into four smaller $T$-cells and one $O$-cell. Each $O$-cell splits into six $O$-cells and eight $T$-cells. At the generic step $\kappa \geq 1$, we get

$$
T \rightarrow \alpha \frac{T}{2^{\kappa}}+\beta \frac{O}{2^{\kappa-1}},
$$

where $\alpha, \beta$ are two positive integers. As a last step, $O$-cells are chopped.

If a tetrahedron $t$ born from this last subdivision is earmarked for refinement by the error-estimator, one must look upward to its ancestry before dividing it. If $t$ is a $T$-cell, apply normal subdivision. Otherwise, backtrack to its mother $O$-cell and subdivide the latter. Apart from those that are $T$-cells, tetrahedra of the subdivision are mules, not able to reproduce by division. The same strategy applies to the transition layer of tetrahedra that touch divided ones, and need division for conformity. They should be cut in two or more tetrahedra, depending on how many of their edges belong to divided tetrahedra. Here, for simplicity, we consider only two cases: a $T$-cell with a divided face results in four tetrahedra and the one with a divided edge results in two. Products of this subdivision can be mules as well as tetrahedra with divided edges or faces and the procedure goes on. All other $T$-cells presenting two or more divided faces are cut according to the normal subdivision (at worst, the normal subdivision applies to the whole set of tetrahedra). If one of the two or four tetrahedra $t$ that compose a $T$-cell in the transition layer is pointed at for subdivision, one backtracks to its (mother) $T$-cell and applies normal subdivision. (Refining tetrahedra $t$ the same way, by the normal subdivision that served for the $T$-cell, would make a mess.) The number of different tetrahedral shapes is thus kept small, whatever the depth of the subdivision procedure. 


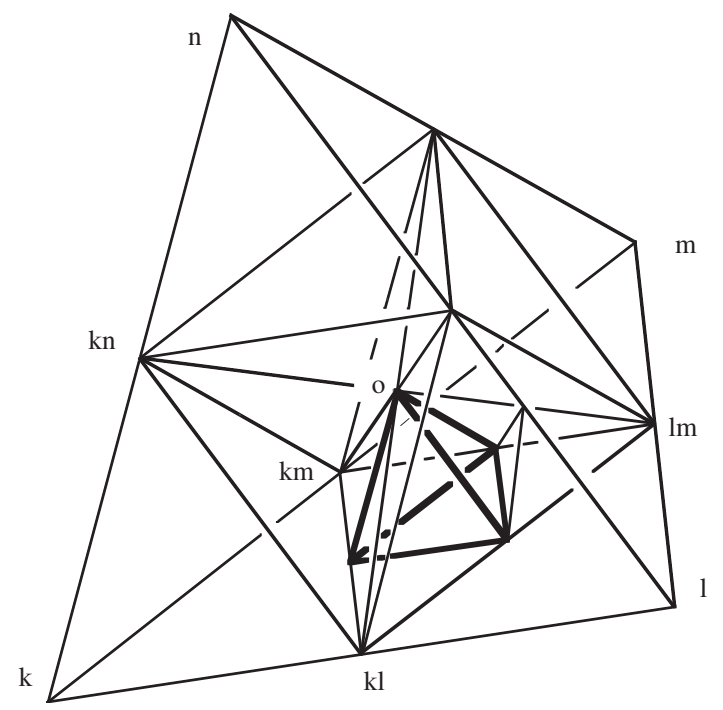

FIG. 5. Normal refinement for the tetrahedron $T=\{k, l, m, n\}$. Mid-edges are denoted $k l, l m$, etc., and $o$ is the barycenter. A first halving of edges generates four small tetrahedra and a core octahedron, which itself can be divided into eight "octants" such as $O=\{o, k l, l m, m k\}$, of at most four different shapes. At this point, we have twelve small tetrahedra, only eight in the octahedron. Now, octants like $O$ should be subdivided as follows: Divide the face in front of o into four triangles and join to o; hence we have a tetrahedron similar to $T$, and three peripheral tetrahedra. These, in turn, are halved as shown for the one hanging from edge $\{o, l m\}$. Its two parts are similar to $O$ and to the neighbor octant $\{o, k n, k l, m k\}$, respectively. At the end of the second step, we have 56 tetrahedra for the core octahedron.

Note that, starting from a given mesh, the barycentric subdivision as well as the normal one do not change the homology group of a complex, since the triangulated domain is always the same. This is the very point of homology (see, for example, [1]).

4. Construction of a restriction/prolongation operator between two nested meshes. Recall that the collection of groups and homomorphisms

$$
\{0\} \longrightarrow \ldots \stackrel{\partial}{\longrightarrow} C_{p}(m) \stackrel{\partial}{\longrightarrow} C_{p-1}(m) \stackrel{\partial}{\longrightarrow} \ldots \longrightarrow\{0\}
$$

is usually referred to as the chain complex of the mesh $m$ and denoted by $C(m)$. Here, we shall consider two meshes, the coarse one $m$ and the fine one $\tilde{m}$, as obtained from $m$ by a given refinement technique; hence we have two complexes $C(m)$ and $C(\tilde{m})$. We use capital letters to denote nodes, edges, faces, and volumes in $m$ and lowercase letters to denote analogous cells in $\tilde{m}$. Incidence matrices for $\tilde{m}$ are denoted $g, r, d$. Recall that the elementary chain associated with a simplex of $m$ (or $\tilde{m}$ ) and the simplex itself are denoted by the same symbol. Last, we shall use the shorthand " $s \subset S$ " when simplex $s$ is, as a subset of the three-dimensional space, a part of $S$. (Thus, $N \subset E$ means $N$ is an endpoint of $E$. In the case of nodes, $n \subset N$ just means that $n$ and $N$ sit at the same point.)

In what follows, we first introduce two maps, $\chi: C(m) \rightarrow C(\tilde{m})$ and $\pi: C(\tilde{m}) \rightarrow$ $C(m)$; we next prove that $\chi$ and $\pi$ are "chain maps," as defined below, and that $\pi \chi=1$. 
Definition 4.1. Given a p-simplex $S$ of the coarse complex $m$, set

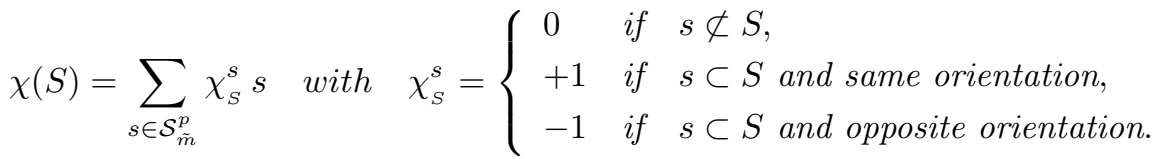

Of course, the map $\chi$ is the natural way to embed $m$ into $\tilde{m}$ : Chop the large simplex into small ones, and build a chain from these, with weights \pm 1 according to respective orientations. For nodes, we assumed positive orientation for all of them, so $\chi_{N}^{n}$ is 1 if $n$ coincides with $N, 0$ otherwise.

Next, let $w^{S}$ denote the Whitney form associated with a $p$-simplex $S$ of the coarse mesh so that $\left\langle w^{S} ; S^{\prime}\right\rangle=\delta_{S, S^{\prime}}$ for all $p$-simplices $S^{\prime} \in m$. Then we have the following definition.

Definition 4.2. Given a p-simplex s of the fine complex $\tilde{m}$, set

$$
\pi(s)=\sum_{S \in \mathcal{S}_{m}^{p}}\left\langle w^{S} ; s\right\rangle S \equiv \sum_{S \in \mathcal{S}_{m}^{p}} \pi_{s}^{S} S .
$$

A small simplex is thus represented by a chain of big ones. (The use of Whitney forms for this is natural: As argued elsewhere [5], Whitney forms are best viewed as a device to represent manifolds by simplicial chains. Here, the manifold is the small simplex s.) We now prove three propositions.

Proposition 4.3. One has $\pi \chi=1$.

Proof. We must show that $\pi(\chi(S))=S$ for any coarse $p$-simplex $S$. Indeed,

$$
\begin{aligned}
\pi(\chi(S)) & =\pi\left(\sum_{s \in \mathcal{S}_{\tilde{m}}^{p}} \chi_{S}^{s} s\right) \\
& =\sum_{s \in \mathcal{S}_{\tilde{m}}^{p}} \chi_{S}^{s} \sum_{S^{\prime} \in \mathcal{S}_{m}^{p}}\left\langle w^{S^{\prime}} ; s\right\rangle S^{\prime} \\
& =\sum_{S^{\prime} \in \mathcal{S}_{m}^{p}}\left\langle w^{S^{\prime}} ; \sum_{s \in \mathcal{S}_{\tilde{m}}^{p}} \chi_{S}^{s} s\right\rangle S^{\prime} \\
& =\sum_{S^{\prime} \in \mathcal{S}_{m}^{p}}\left\langle w^{S^{\prime}} ; S\right\rangle S^{\prime} \\
& =S
\end{aligned}
$$

thanks to the fundamental property of Whitney forms, $\left\langle w^{S^{\prime}} ; S\right\rangle=\delta_{S, S^{\prime}}$. To pass from (6) to (7), use the equality $\langle w ; S\rangle=\langle w ; \chi(S)\rangle$, for any given $p$-form $w$, which stems from additivity of the integral.

It is important to remark that Proposition 4.3 and its proof do not depend on the refinement technique, but just on the fact that coarse cells are tessellations of small ones.

Proposition 4.4. The map $\chi$ defined in (4) is a chain map, i.e., $\partial \chi=\chi \partial$.

Proof. Although both statement and proof are independent of the refinement procedure, we suppose here that the normal subdivision is considered at the first step (where each tetrahedron gives 12 small tetrahedra) to build up the fine complex $\tilde{m}$ 
from the coarse one $m$. We also treat separately the cases $p=1,2,3$, where $p$ is the dimension of the chain on which $\partial \chi$ and $\chi \partial$ act, though as will be apparent a generic proof (much shorter, but perhaps less informative) could be given. Our purpose is to help understand, on concrete examples, what is going on.

For $p=1$, we have

$$
\begin{aligned}
\chi \partial E & =\chi\left(\sum_{N \in \mathcal{N}_{m}} G_{E, N} N\right) \\
& =\sum_{N \in \mathcal{N}_{m}} G_{E, N} \sum_{n \in \mathcal{N}_{\tilde{m}}} \chi_{N}^{n} n \\
& =\sum_{n \in \mathcal{N}_{\tilde{m}}}\left[\sum_{N \in \mathcal{N}_{m}} G_{E, N} \chi_{N}^{n}\right] n=\sum_{n \in \mathcal{N}_{\tilde{m}}}\left[\sum_{N \subset E} G_{E, N} \chi_{N}^{n}\right] n,
\end{aligned}
$$

since only those nodes $N$ that are, as sets, part of $E$, make $G_{E, N} \neq 0$, and thus contribute to the sum. On the other hand, we obtain:

$$
\begin{aligned}
\partial \chi E & =\partial\left(\sum_{e \in \mathcal{E}_{\tilde{m}}} \chi_{E}^{e} e\right) \\
& =\sum_{e \in \mathcal{E}_{\tilde{m}}} \chi_{E}^{e} \sum_{n \in \mathcal{N}_{\tilde{m}}} g_{e, n} n \\
& =\sum_{n \in \mathcal{N}_{\tilde{m}}}\left[\sum_{e \in \mathcal{E}_{\tilde{m}}} \chi_{E}^{e} g_{e, n}\right] n=\sum_{n \in \mathcal{N}_{\tilde{m}}}\left[\sum_{e \subset E} \chi_{E}^{e} g_{e, n}\right] n,
\end{aligned}
$$

since only those $e$ that are, as sets, part of $E$, make $\chi_{E}^{e} \neq 0$, and thus contribute to the sum. The conclusion comes from the equality between bracketed terms above, which stems from the interplay between incidence numbers on both meshes, as we now show in detail.

If $n \not \subset E$, there is no $N$ such that $n \subset N \subset E$, so the first bracket vanishes. There is no $e$ either such that $n \subset e \subset E$, so the second bracket vanishes too. Assuming therefore $n \subset E$, we have two cases to examine, illustrated by the center part and the right-hand part of Figure 6, where $E$ is supposed to be $E_{2}$ : either $n \subset N$ for $N$ one of the endpoints of $E_{2}$ (say $N_{1}$ or $N_{2}$ ), or not (see Figure 6 , center and right-hand part, respectively).

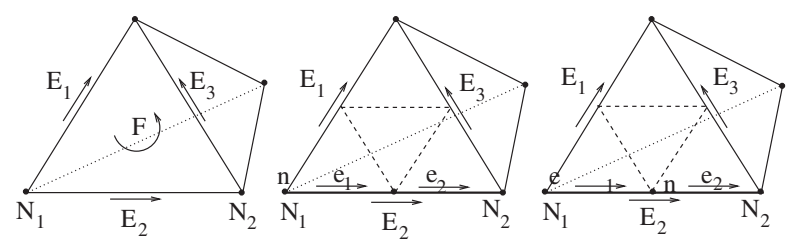

FIG. 6. For node $n \in \mathcal{N}_{\tilde{m}}$ belonging to edge $E_{2}$, either there exists $N \subset E_{2}$ such that $n \subset N$ (center) or not (right).

According to the situation at the center of Figure $6\left(n \subset N_{1}\right)$, we have

$$
\sum_{N \subset E_{2}} G_{E_{2}, N} \chi_{N}^{n}=G_{E_{2}, N_{1}} \chi_{N_{1}}^{n}+G_{E_{2}, N_{2}} \chi_{N_{2}}^{n}=(-1)(1)+(1)(0)=-1 .
$$

For the situation at the right-hand side of Figure 6 , since $n \not \subset N_{i}$, for $i=1$ or 2 , we have

$$
\chi_{N_{i}}^{n}=0 \quad \forall i \quad \text { so that } \sum_{N \subset E} G_{E, N} \chi_{N}^{n}=0 .
$$

Let us do the same reasoning for the other quantity, looking at Figure 6. For the situation at the center of Figure 6, we have (recalling that $g$ is the "fine" incidence 
matrix)

$$
\sum_{e \subset E_{2}} g_{e, n} \chi_{E_{2}}^{e}=g_{e_{1}, n} \chi_{E_{2}}^{e_{1}}+g_{e_{2}, n} \chi_{E_{2}}^{e_{2}}=(-1)(1)+(0)(1)=-1
$$

For the situation at the right-hand side of Figure 6, we have

$$
\sum_{e \subset E_{2}} g_{e, n} \chi_{E_{2}}^{e}=g_{e_{1}, n} \chi_{E_{2}}^{e_{1}}+g_{e_{2}, n} \chi_{E_{2}}^{e_{2}}=(1)(1)+(-1)(1)=0
$$

Summing up, for a given $n \in \mathcal{N}_{\tilde{m}}$, the two quantities in brackets take the same value $(-1,1$ or 0$)$, due to the definition of the incidence matrices $G$ and $g$ and coefficients $\chi_{N}^{n}$ and $\chi_{E}^{e}$.

For $p=2$, we can write that

$$
\begin{aligned}
\chi \partial F & =\chi\left(\sum_{E \in \mathcal{E}_{m}} R_{F, E} E\right) \\
& =\sum_{E \in \mathcal{E}_{m}} R_{F, E} \sum_{e \in \mathcal{E}_{\tilde{m}}} \chi_{E}^{e} e \\
& =\sum_{e \in \mathcal{E}_{\tilde{m}}}\left[\sum_{E \in \mathcal{E}_{m}} R_{F, E} \chi_{E}^{e}\right] e=\sum_{e \in \mathcal{E}_{\tilde{m}}}\left[\sum_{E \subset F} R_{F, E} \chi_{E}^{e}\right] e .
\end{aligned}
$$

On the other hand, we have

$$
\begin{aligned}
& \partial \chi F=\partial\left(\sum_{f \in \mathcal{F}_{\tilde{m}}} \chi_{F}^{f} f\right) \\
& =\sum_{f \in \mathcal{F}_{\tilde{m}}} \chi_{F}^{f} \sum_{e \in \mathcal{E}_{\tilde{m}}} r_{f, e} e \\
& =\sum_{e \in \mathcal{E}_{\tilde{m}}}\left[\sum_{f \in \mathcal{F}_{\tilde{m}}} \chi_{F}^{f} r_{f, e}\right] e=\sum_{e \in \mathcal{E}_{\tilde{m}}}\left[\sum_{f \subset F} \chi_{F}^{f} r_{f, e}\right] e .
\end{aligned}
$$

We compare again the two quantities in brackets, noting again that both brackets vanish for each $e \in \mathcal{E}_{\tilde{m}}$ such that $e \not \subset F$. Assuming therefore $e \subset F$ (Figure 7), we have two cases: Either there exists $E \in \mathcal{E}_{m}$ such that $e \subset E$ and $E \subset F$, or not (see Figures 7 and 8, center and right-hand part, respectively).
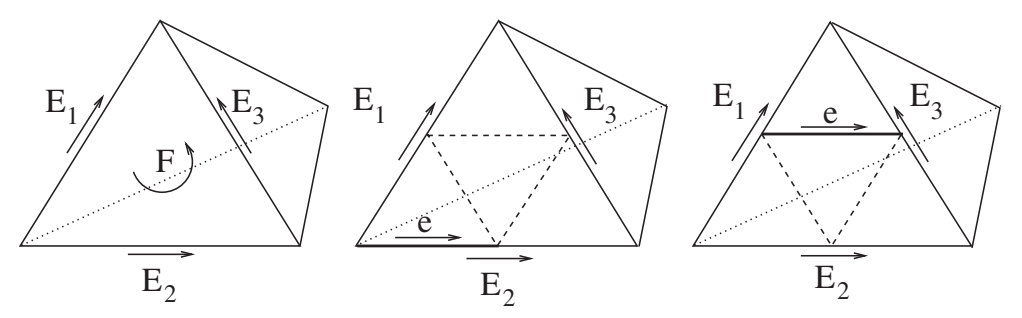

FIG. 7. For edge $e \in \mathcal{E}_{\tilde{m}}$ belonging to face $F$, either there exists $E \subset F$ such that $e \subset E$ (center) or not (right).

According to the situation at the center of Figure 7, we have

$$
\begin{aligned}
\sum_{E \subset F} R_{F, E} \chi_{E}^{e} & =R_{F, E_{1}} \chi_{E_{1}}^{e}+R_{F, E_{2}} \chi_{E_{2}}^{e}+R_{F, E_{3}} \chi_{E_{3}}^{e} \\
& =(-1)(0)+(1)(1)+(1)(0)=1 .
\end{aligned}
$$

For the situation at the right-hand side of Figure 7 , since $e \not \subset E_{i}, i=1,2,3$, we have

$$
\chi_{E_{i}}^{e}=0 \quad \forall i \quad \text { so that } \sum_{E \subset F} R_{F, E} \chi_{E}^{e}=0 .
$$



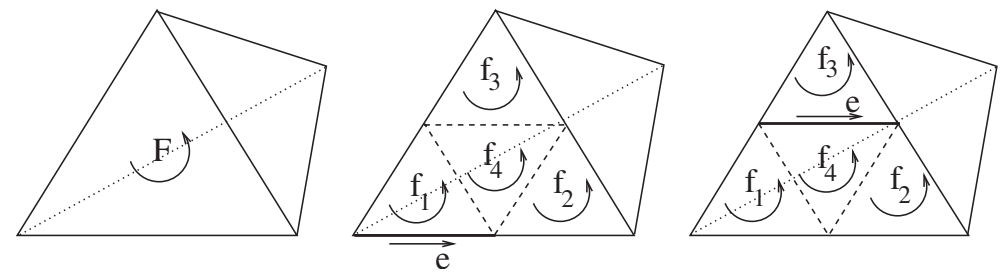

FIG. 8. For edge e $\in \mathcal{E}_{\tilde{m}}$ belonging to face $F$, either there exists only one face $f \in \mathcal{E}_{\tilde{m}}$ such that $e \subset f$ (center) or two (right).

Let us do the same reasoning for the other quantity, looking at Figure 8. For the situation at the center of Figure 8, we have

$$
\begin{aligned}
\sum_{f \subset F} \chi_{F}^{f} r_{f, e} & =\chi_{F}^{f_{1}} r_{f_{1}, e}+\chi_{F}^{f_{2}} r_{f_{2}, e}+\chi_{F}^{f_{3}} r_{f_{3}, e}+\chi_{F}^{f_{4}} r_{f_{4}, e} \\
& =(1)(1)+(0)(1)+(0)(1)+(0)(1)=1 .
\end{aligned}
$$

For the situation at the right-hand side of Figure 8, we have

$$
\begin{aligned}
\sum_{f \subset F} r_{f, e} \chi_{F}^{f} & =\chi_{F}^{f_{1}} r_{f_{1}, e}+\chi_{F}^{f_{2}} r_{f_{2}, e}+\chi_{F}^{f_{3}} r_{f_{3}, e}+\chi_{F}^{f_{4}} r_{f_{4}, e} \\
& =(0)(1)+(0)(1)+(1)(1)+(-1)(1)=0 .
\end{aligned}
$$

Summing up, for a given $e \in \mathcal{E}_{\tilde{m}}$, the two quantities in brackets take the same value ( 1 or 0 ), due to the definition of the incidence matrices $R$ and $r$ and coefficients $\chi_{E}^{e}$ and $\chi_{F}^{f}$.

Finally, for $p=3$, we have

$$
\begin{aligned}
\chi \partial T & =\chi\left(\sum_{F \in \mathcal{F}_{m}} D_{T, F} F\right) \\
& =\sum_{F \in \mathcal{F}_{m}} D_{T, F} \sum_{f \in \mathcal{F}_{\tilde{m}}} \chi_{F}^{f} f \\
& =\sum_{f \in \mathcal{F}_{\tilde{m}}}\left(\sum_{F \in \mathcal{F}_{m}} D_{T, F} \chi_{F}^{f}\right) f=\sum_{f \in \mathcal{F}_{\tilde{m}}}\left[\sum_{F \subset T} D_{T, F} \chi_{F}^{f}\right] f .
\end{aligned}
$$

On the other hand,

$$
\begin{aligned}
\partial \chi T & =\partial\left(\sum_{t \in \mathcal{T}_{\tilde{m}}} \chi_{T}^{t} t\right) \\
& =\sum_{t \in \mathcal{T}_{\tilde{m}}} \chi_{T}^{t} \sum_{f \in \mathcal{F}_{\tilde{m}}} d_{t, f} f \\
& =\sum_{f \in \mathcal{F}_{\tilde{m}}}\left(\sum_{t \in \mathcal{T}_{\tilde{m}}} \chi_{T}^{t} d_{t, f}\right) f=\sum_{f \in \mathcal{F}_{\tilde{m}}}\left[\sum_{t \subset T} \chi_{T}^{t} d_{t, f}\right] f .
\end{aligned}
$$

We compare again the two quantities in brackets, assuming $f \subset T$. We have two cases: either there exists $F \in \mathcal{F}_{m}$ such that $f \subset F$ or not (see Figure 9's left-hand and right-hand part, respectively). If $f$ is part of, say, $F_{1}$, then

$$
\begin{aligned}
\sum_{F \subset T} D_{T, F} \chi_{F}^{f} & =D_{T, F_{1}} \chi_{F_{1}}^{f}+D_{T, F_{2}} \chi_{F_{2}}^{f}+D_{T, F_{3}} \chi_{F_{3}}^{f}+D_{T, F_{4}} \chi_{F_{4}}^{f} \\
& =(1)(1)+(1)(0)+(1)(0)+(1)(0)=1 .
\end{aligned}
$$

If $f \not \subset F_{i}$, whatever $F_{i} \subset T$, then

$$
\chi_{F_{i}}^{f}=0 \quad \forall i ; \quad \text { hence } \quad \sum_{F \subset T} D_{T, F} \chi_{F}^{f}=0 .
$$

For the other bracketed term, either $f$ is part of some $F_{1}$, and there exists only one $t \in \mathcal{T}_{\tilde{m}}$ containing $f$, namely $t^{*}$, so that

$$
\sum_{t \subset T} d_{t, f} \chi_{T}^{t}=d_{t^{*}, f} \chi_{T}^{t^{*}}=(1)(1)=1
$$



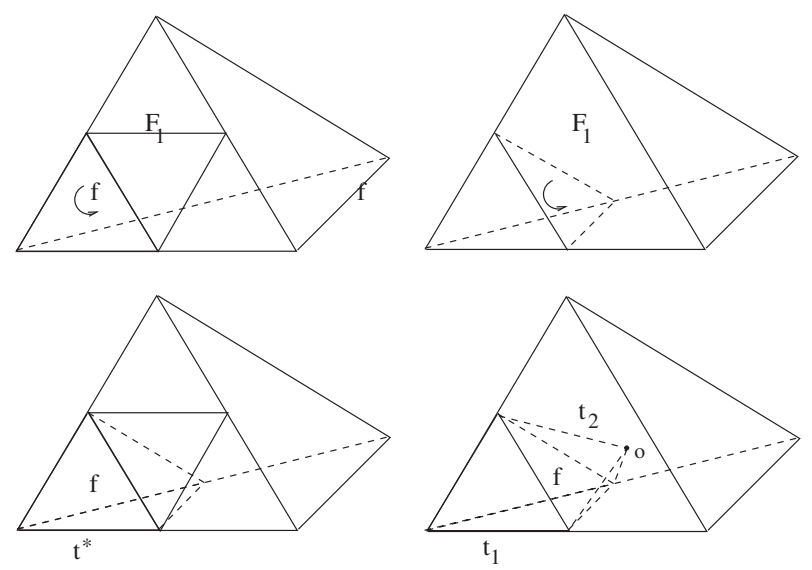

Fig. 9. For face $f \in \mathcal{F}_{\tilde{m}}$ belonging to tetrahedron $T \in \mathcal{T}_{m}$, either there exists $F \subset T$ such that $f \subset F$ (left) or not (right). In the first case, there exists a unique tetrahedron $t^{*} \in \mathcal{T}_{\tilde{m}}$ containing $f$, and in the second case, two tetrahedra $t_{1}, t_{2} \in \mathcal{T}_{\tilde{m}}$. The normal subdivision of $T$ is not completely shown to make the figure clearer (o is the barycenter of $T$ ).

or $f \not \subset F_{i}$ whatever $F_{i} \subset T$. Then, $f$ is inside $T$ and is thus shared by two tetrahedra of $\tilde{m}$, say $t_{1}$ and $t_{2}$. So,

$$
\sum_{t \subset T} d_{t, f} \chi_{T}^{t}=d_{t_{1}, f} \chi_{T}^{t_{1}}+d_{t_{2}, f} \chi_{T}^{t_{2}}=(1)(1)+(-1)(1)=0 .
$$

Summing up, for a given $f \in \mathcal{F}_{\tilde{m}}$, the two quantities in brackets take the same value ( 1 or 0 ), owing to the definition of the incidence matrices $D$ and $d$ and coefficients $\chi_{F}^{f}$ and $\chi_{T}^{t}$.

This completes the proof, which has been detailed for all cases, much beyond logical necessity, to show how the incidence matrices and the two maps interact. Later on, we will consider only one case, the others being on the same pattern.

Proposition 4.5. The map $\pi$ defined in (5) is a chain map, i.e., $\partial \pi=\pi \partial$.

Proof. We consider the case $p=2$ to detail the proof. Then

$$
\begin{aligned}
\pi \partial f & =\pi\left(\sum_{e \in \mathcal{E}_{\tilde{m}}} r_{f, e} e\right) \\
& =\sum_{e \in \mathcal{E}_{\tilde{m}}} r_{f, e} \sum_{E \in \mathcal{E}_{m}}\left\langle w^{E} ; e\right\rangle E \\
& =\sum_{E \in \mathcal{E}_{m}}\left[\sum_{e \in \mathcal{E}_{\tilde{m}}} r_{f, e}\left\langle w^{E} ; e\right\rangle\right] E .
\end{aligned}
$$

On the other hand, we can write

$$
\begin{aligned}
\partial \pi f & =\partial\left(\sum_{F \in \mathcal{F}_{m}}\left\langle w^{F} ; f\right\rangle F\right) \\
& =\sum_{F \in \mathcal{F}_{m}}\left\langle w^{F} ; f\right\rangle \sum_{E \in \mathcal{E}_{m}} R_{F, E} E \\
& =\sum_{E \in \mathcal{E}_{m}}\left[\sum_{F \in \mathcal{F}_{m}} R_{F, E}\left\langle w^{F} ; f\right\rangle\right] E .
\end{aligned}
$$

We now recall that, when $p=2$,

$$
\mathrm{d} w^{E}=\sum_{F \in \mathcal{F}_{m}} R_{F, E} w^{F}
$$


so that

$$
\begin{aligned}
\partial \pi f & =\sum_{E \in \mathcal{E}_{m}}\left[\sum_{F \in \mathcal{F}_{m}} R_{F, E}\left\langle w^{F} ; f\right\rangle\right] E \\
& =\sum_{E \in \mathcal{E}_{m}}\left[\left\langle\mathrm{~d} w^{E} ; f\right\rangle\right] E \\
& =\sum_{E \in \mathcal{E}_{m}}\left[\left\langle w^{E} ; \partial f\right\rangle\right] E \\
& =\sum_{E \in \mathcal{E}_{m}}\left[\sum_{e \in \mathcal{E}_{\tilde{m}}} r_{f, e}\left\langle w^{E} ; e\right\rangle\right] E \\
& =\pi \partial f .
\end{aligned}
$$

Note the two ingredients of the proof: the Stokes theorem and the structural property, (8), of the Whitney complex. For other dimensions, the proof is similar: Just change $R$ and $r$ into $G$ and $g$ if $p=1$, into $D$ and $d$ if $p=3$.

Remark 4.6. The chain map $\chi: C(m) \rightarrow C(\tilde{m})$ can be defined, similarly to $\pi$, as follows: Given a $p$-simplex $S$ of the coarse complex $m$, set

$$
\chi(S)=\sum_{s \in \mathcal{S}_{\tilde{m}}^{p}}\left\langle w^{s} ; S\right\rangle s=\sum_{s \in \mathcal{S}_{\tilde{m}}^{p}} \chi_{S}^{s} s .
$$

In the nested case, definitions (9) and (4) coincide. In the nonnested case, (9) is a generalization of (4); the property $\pi \chi=1$ is lost, and the coefficients $\pi_{s}^{S}$ and $\chi_{S}^{s}$ cannot be computed "by hands" as we shall see in the next section for nested grids.

5. Application. We explain how the map $\pi$ can be used to design a multigrid algorithm for the solution of linear systems arising from the use of Whitney elements on tetrahedra to discretize a given differential (e.g., electromagnetic) problem. The detailed analysis of the mesh-independent convergence and performances of the multigrid algorithm based on $\pi$ is not considered here. We refer to $[3,8,14]$ for rigorous theoretical and numerical results in the edge element framework, and to [6] for a formulation and application of the multigrid algorithm on hexahedral meshes.

As already pointed out in the introduction, the motivation for this approach comes from the analysis of the error on the numerical solution in the frequency domain. We recall the basic multigrid algorithm, assuming a two-grid method for simplicity. Let $h$ and $H$ denote, respectively, the maximal diameter of tetrahedra in the fine $\tilde{m}$ and coarse $m$ grids. Let $\mathcal{V}_{h}$ and $\mathcal{V}_{H}$ be the underlying finite dimensional spaces of cochains, with $\operatorname{dim}\left(\mathcal{V}_{h}\right)>\operatorname{dim}\left(\mathcal{V}_{H}\right)$, consistent with $h<H$. One wishes to solve the linear system $A_{h} \mathbf{u}_{h}=\mathbf{b}_{h}$ in $\mathcal{V}_{h}$, assuming that the matrix $A_{h}$ is symmetric and positive definite (as is usually the case for matrices resulting from finite element discretizations of a variational problem). Denoted by $(\mathbf{u}, \mathbf{v})$ the scalar product of $\mathbf{u}, \mathbf{v} \in \mathcal{V}_{h}$, solving $A_{h} \mathbf{u}_{h}=\mathbf{b}_{h}$ in $\mathcal{V}_{h}$ is then equivalent to finding the minimizer $\mathbf{u}_{h} \in \mathcal{V}_{h}$ of the quadratic functional $\Phi(\mathbf{u})=\frac{1}{2}\left(A_{h} \mathbf{u}, \mathbf{u}\right)-\left(\mathbf{b}_{h}, \mathbf{u}\right)$. In what follows, $M_{h}$ represents a suitable preconditioner for $A_{h}$. The maps $R_{h}^{H}: \mathcal{V}_{h} \rightarrow \mathcal{V}_{H}$, usually called restriction operator, and $P_{H}^{h}: \mathcal{V}_{H} \rightarrow \mathcal{V}_{h}$, called prolongation operator, are full-rank linear cochain-to-cochain operators. The so-called two-level $V$-cycle of the multigrid procedure reads as follows: 
1. Fine grid presmoothing: from $\mathbf{u}_{h}^{0} \in \mathcal{V}_{h}$ and for $k=1, \ldots, n_{1}$, do

$$
\mathbf{u}_{h}^{k}=\mathbf{u}_{h}^{k-1}+M_{h}\left(\mathbf{b}_{h}-A_{h} \mathbf{u}_{h}^{k-1}\right) .
$$

2. Coarse grid correction: given $\mathbf{r}_{h}^{n_{1}}=\mathbf{b}_{h}-A_{h} \mathbf{u}_{h}^{n_{1}}$ in $\mathcal{V}_{h}$, do

restrict the residual on the coarse grid: $\mathbf{r}_{H} \leftarrow R_{h}^{H} \mathbf{r}_{h}^{n_{1}}$.

solve the residual problem: $A_{H} \mathbf{z}_{H}=\mathbf{r}_{H}$.

correct the solution in $\mathcal{V}_{h}: \mathbf{u}_{h}^{n_{1}} \leftarrow \mathbf{u}_{h}^{n_{1}}+P_{H}^{h} \mathbf{z}_{H}$.

3. Fine grid postsmoothing: from $\mathbf{u}_{h}^{n_{1}} \in \mathcal{V}_{h}$ and for $k=1, \ldots n_{2}$, do (10).

In the fine grid presmoothing step, one iteratively solves $A_{h} \mathbf{u}_{h}=\mathbf{b}_{h}$ in $\mathcal{V}_{h}$ by a basic iterative method. High frequency errors are thus well eliminated, and once this is achieved in, e.g., $n_{1}$ iterations, further fine grid iterations would not improve significantly the convergence rate. In the coarse grid correction, one tries to correct $\mathbf{u}_{h}^{n_{1}}$ on the coarse space $\mathcal{V}_{H}$. The coarse correction $\mathbf{z}_{H}$ minimizes $\Phi\left(\mathbf{u}_{h}^{n_{1}}+P_{H}^{h} \mathbf{z}_{H}\right)$ over $\mathcal{V}_{H}$. This is equivalent to solving $\left(P_{H}^{h}\right)^{t} A_{h} P_{H}^{h} \mathbf{z}_{H}=\left(P_{H}^{h}\right)^{t} \mathbf{r}_{h}^{n_{1}}$ on $\mathcal{V}_{H}$. Thus, $A_{H}=\left(P_{H}^{h}\right)^{t} A_{h} P_{H}^{h}$ and the $R_{h}^{H}$ of step 2 is the transpose $\left(P_{H}^{h}\right)^{t}$ [16]. On $m$, the low frequency errors of $\tilde{m}$ manifest themselves as relatively high frequency errors and are thus eliminated efficiently using again simple iterative smoothing methods. If the coarsest grid has been reached, the coarse system has to be solved exactly, by a direct solver, which can be done with little computational effort due to the small number of unknowns. Otherwise, the three-step multigrid procedure can be repeated recursively to solve the residual problem, as many times as the number of coarsening levels $m$ one considers, starting from the fine one $\tilde{m}$. Each grid level is responsible for eliminating a particular frequency bandwidth of the error. Finally, in the fine grid postsmoothing step, one solves iteratively $n_{2}$ times in $\mathcal{V}_{h}$ the system $A_{h} \mathbf{u}_{h}=\mathbf{b}_{h}$, starting from $\mathbf{u}_{h}^{n_{1}}+P_{H}^{h} \mathbf{z}_{H}$, to eliminate high frequency errors on the term $P_{H}^{h} \mathbf{z}_{H}$.

Our proposal is now to define the operator $P_{H}^{h}$ as the dual of the chain map $\pi$. Indeed, recall that $\mathcal{V}_{h}$ and $\mathcal{V}_{H}$ are spaces of $p$-cochains, while $\pi$ is defined on $p$-chains (see Definition 4.2). There is therefore a natural prolongation operator $P_{H}^{h}$, defined as the dual of $\pi$, i.e., by $\langle\mathbf{u} ; \pi(s)\rangle=\left\langle P_{H}^{h} \mathbf{u} ; s\right\rangle$ for all $p$-chains $s$ and $p$-cochains $\mathbf{u} \in \mathcal{V}_{H}$, as suggested by the diagram below.

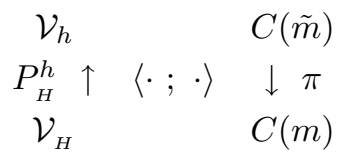

Taking dual bases on both $\mathcal{V}_{h}$ and $\mathcal{V}_{H}$ as explained in section 2.3, the matrix representation of $P_{H}^{h}$ has entries $\left(P_{H}^{h}\right)_{S}^{S}=\pi_{S}^{S}$ (cf. (5)). Recall that $S$ and $s$ here are two simplices of same dimension $p$ in $m$ and $\tilde{m}$, respectively, so that there are distinct prolongation operators for each $p$, i.e., for degrees of freedom based on nodes, edges, faces, and volumes.

We now detail the calculation of $\pi$ in the case where a number of coarse tetrahedra have undergone one normal subdivision, i.e., by using (3) with $\kappa=1$ (see Figure 5), thus being divided into twelve small ones, while tetrahedra in the transition layer are split into four or two small ones; hence we have the three cases considered below. It is important to remark that the chain-map coefficients $\pi_{s}^{S}$ we search, defined as $\left\langle w^{S} ; s\right\rangle$ in (5), do not depend on the shape of $S$ and $s$ but on their relative position and orientation. Their computation relies on the following two obvious lemmas. 
Lemma 5.1. Let $s$ be a p-simplex and $w$ a linear $p$-differential form, linear with respect to position $x$. Then $\int_{s} w=\int_{s} w\left(\mathbf{x}_{s}\right)$, where $\mathbf{x}_{s}$ denotes the barycenter of $s$.

This replaces a linear differential form by a constant one. For these, one has:

LEMma 5.2. Let $s$ be a p-simplex and $w$ a constant $p$-differential form, $L$ a linear map which sends simplex $s$ to simplex $s^{\prime}$. Then $\int_{s^{\prime}} w=\operatorname{det}(L) \int_{s} w$.

Case I. Tetrahedron $T$ has been divided into twelve small tetrahedra $t$.

For $p=3$, let $w^{T}$ be the scalar function associated to $T$ (section 2.1), that is the constant such that $\left\langle w^{T} ; T\right\rangle=1$. Computing $\left\langle w^{T} ; t\right\rangle$ thus amounts to finding the relative volume of $t$ (an affine notion, not a metric one) with respect to $T$. This is $\frac{1}{8}$ for the four tetrahedra $t$ sharing a vertex with $T$ (scaling factor $\frac{1}{2}$, to the cube), which leaves $\frac{1}{2}$ to be shared equally between the 8 tetrahedra with a vertex at the barycenter $o$ of $T$. So one has

$$
\begin{array}{ll}
\left\langle w^{T} ; t\right\rangle= \pm \frac{1}{8} & \text { for all } t \text { not contained in the core octahedron; } \\
\left\langle w^{T} ; t\right\rangle= \pm \frac{1}{16} & \text { for all } t \text { contained in the core octahedron. }
\end{array}
$$

The sign \pm 1 depends on the relative orientation between $t$ and $T$.

Note that the Lebesgue measure of $t$ or of $T$ played no role here: Considerations of scaling and symmetry suffice to do the job, as will also be the case for other values of $p$. We give only the results without further comments. Only the nonzero coefficients are displayed.

For $p=2$, there are four different situations, depending on where the small face $f$ is located with respect to the big one $F$ and we refer again to Figure $5, \kappa=1$. The coefficients $\left\langle w^{F} ; f\right\rangle$ are the fluxes of the vector function $w^{F}$ across the small faces $f$. Let $F$ be $\{k, l, n\}$ for definiteness. Using $\left\langle w^{F} ; F\right\rangle=1$, scaling, and symmetry, then

$$
\begin{array}{ll}
\left\langle w^{F} ; f\right\rangle= \pm \frac{1}{4} & \text { for all } f \subset F \text { such as } f=\{k, k l, k n\} ; \\
\left\langle w^{F} ; f\right\rangle= \pm \frac{1}{8} & \text { for all } f \not \subset F \text { and with three vertices at mid-points } \\
& \text { not in } F, \text { such as } f=\{m k, l m, m n\} ; \\
\left\langle w^{F} ; f\right\rangle= \pm \frac{1}{8} & \text { for all } f \not \subset F \text { and with three vertices at mid-points } \\
& \text { and one edge on } F, \text { such as } f=\{k l, k n, k m\} ; \\
\left\langle w^{F} ; f\right\rangle= \pm \frac{1}{16} & \text { for all } f \not \subset F \text { and with two vertices at mid-points } \\
& \text { and the third one at } o \text {, such as } f=\{k l, k n, o\} .
\end{array}
$$

For $p=1$, the coefficients $\left\langle w^{E} ; e\right\rangle$ are the circulations of the vector function $w^{E}$ along the small edges $e$. Consider $E=\{k, n\}$. Again, $\left\langle w^{E} ; E\right\rangle=1$, scaling and symmetry yield

$$
\begin{aligned}
& \left\langle w^{E} ; e\right\rangle= \pm \frac{1}{2} \quad \text { for all } e \subset E \text {, such as } e=\{k, k n\} \text {; } \\
& \left\langle w^{E} ; e\right\rangle= \pm \frac{1}{4} \quad \text { for all } e \not \subset E \text { with vertices at mid-points, } \\
& \text { one of which belongs to } E \text {, such as } e=\{k n, k l\} \text {; } \\
& \left\langle w^{E} ; e\right\rangle= \pm \frac{1}{4} \quad \text { for all } e \not \subset E \text { with vertices at mid-points and } \\
& \text { parallel to } E \text {, such as } e=\{k l, \ln \} \text {; } \\
& \left\langle w^{E} ; e\right\rangle= \pm \frac{1}{8} \quad \text { for all } e \not \subset E \text { with one vertex at } o \text { and one at a mid-point } \\
& \text { not in } E \text {, such as } e=\{l n, o\} \text { or } e=\{m n, o\} \text {. }
\end{aligned}
$$

For $p=0$, the coefficient $\left\langle w^{N} ; n\right\rangle$ is the value of the scalar function $w^{N}$ at node 
$n$. Take for example $N=k$. Using $\left\langle w^{N} ; N\right\rangle=1$ and linearity, one gets

$$
\begin{array}{ll}
\left\langle w^{N} ; n\right\rangle=1 & \text { for } n \subset N ; \\
\left\langle w^{N} ; n\right\rangle=\frac{1}{2} & \text { for } n \text { at the middle of an edge } \\
& \text { incident on } N, \text { such as } k n \text { or } k m ; \\
\left\langle w^{N} ; n\right\rangle=\frac{1}{4} & \text { for } n \text { at } o .
\end{array}
$$

Case II. Here, $T$ is a tetrahedron of the transition layer, divided into four small tetrahedra $t$, as shown in Figure 10 (left and right).
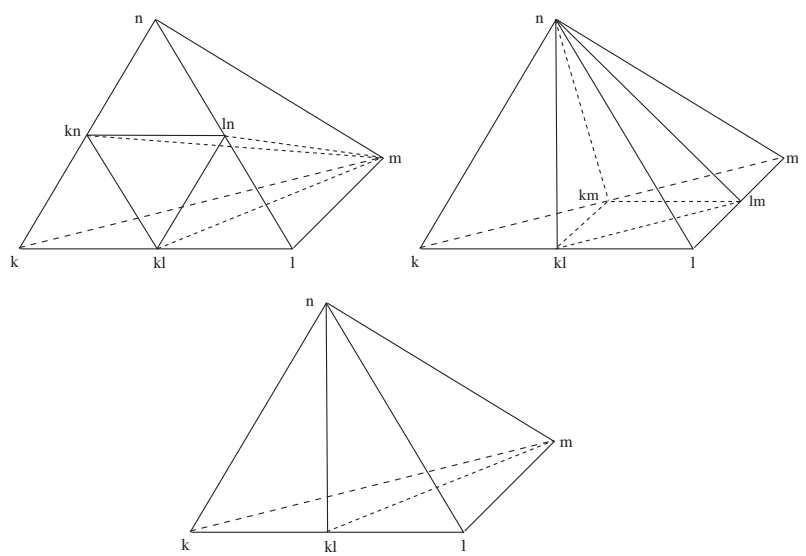

FIG. 10. Tetrahedron $T$ in the transition layer and division in four and two tetrahedra $t$.

For $p=3$, by symmetry, $\left\langle w^{T} ; t\right\rangle= \pm \frac{1}{4} \quad$ for all $t$ contained in $T$.

For $p=2$, two cases. If $F$ (taken here as $\{k, l, n\}$ for the sake of the example) is divided in four as in Figure 10 (left), then

$$
\left\langle w^{F} ; f\right\rangle= \pm \frac{1}{4} \quad \text { for all } f \subset F \text {, such as } f=\{k, k l, k n\},
$$

while $F$ is divided in two, as in Figure 10 (right), then

$$
\begin{array}{ll}
\left\langle w^{F} ; f\right\rangle= \pm \frac{1}{2} & \text { for all } f \subset F, \text { such as } f=\{k, k l, n\} ; \\
\left\langle w^{F} ; f\right\rangle= \pm \frac{1}{4} & \text { for all } f \text { neither in } F \text { nor in } F^{\prime} \neq F, \text { such as } f=\{k l, k m, n\} .
\end{array}
$$

For $p=1$, two cases again. If $E \subset F$ and $F$ is divided in four, then (with $E=\{k, n\}$ for illustration)

$$
\begin{array}{ll}
\left\langle w^{E} ; e\right\rangle= \pm \frac{1}{2} & \text { for all } e \subset E, \text { such as } e=\{k, k n\} ; \\
\left\langle w^{E} ; e\right\rangle= \pm \frac{1}{4} & \text { for all } e \not \subset E \text { with vertices at mid-points, } \\
& \text { one of which belongs to } E, \text { such as } e=\{k n, k l\} ;
\end{array}
$$

$$
\begin{array}{ll}
\left\langle w^{E} ; e\right\rangle= \pm \frac{1}{4} & \text { for all } e \not \subset E \text { parallel to } E \text { with } \\
& \text { vertices at mid-points, such as } e=\{k l, l n\},
\end{array}
$$

while if $F$ is divided in two, then 
$\begin{array}{ll}\left\langle w^{E} ; e\right\rangle=1 & \text { for } e=E ; \\ \left\langle w^{E} ; e\right\rangle= \pm \frac{1}{2} & \text { for } e=\{k l, n\} \text { and }\{k m, n\} .\end{array}$

For $p=0$, by linearity,

$$
\begin{array}{ll}
\left\langle w^{N} ; n\right\rangle=1 & \text { for } n \text { at } N ; \\
\left\langle w^{N} ; n\right\rangle=\frac{1}{2} & \begin{array}{l}
\text { for all } n \text { mid-points of edges with one } \\
\text { extremity at } N, \text { such as } k n \text { or } k l .
\end{array}
\end{array}
$$

Case III. Now $T$, is halved, as shown in Figure 10 (bottom).

For $p=3, \quad\left\langle w^{T} ; t\right\rangle= \pm \frac{1}{2} \quad$ for all $t$ contained in $T$.

For $p=2$ and $F$ halved (as in Figure 10, bottom, take $F=\{k, l, n\}$ for illustration),

$$
\left\langle w^{F} ; f\right\rangle= \pm \frac{1}{2} \quad \text { for all } f \subset F \text {, such as } f=\{k, k l, n\} .
$$

If $F^{\prime} \neq F$ is halved, then

$$
\begin{array}{ll}
\left\langle w^{F} ; f\right\rangle=1 & \text { for } f \equiv F ; \\
\left\langle w^{F} ; f\right\rangle= \pm \frac{1}{2} & \text { for } f=\{k, l m, n\} .
\end{array}
$$

For $p=1$, if $E \subset F$ and $F$ is divided in two but not $E$, then (consider $E=\{k, n\}$ )

$$
\begin{array}{ll}
\left\langle w^{E} ; e\right\rangle=1 & \text { for } e=E ; \\
\left\langle w^{E} ; e\right\rangle= \pm \frac{1}{2} & \text { for } e=\{k l, n\} .
\end{array}
$$

If $E \subset F$ and $F$ is not divided in two, then

$$
\left\langle w^{E} ; e\right\rangle=1 \quad \text { for } e=E
$$

and last, if $E \subset F$ and $F$ is divided in two along $E$, then

$$
\begin{aligned}
\left\langle w^{E} ; e\right\rangle & = \pm \frac{1}{2} \quad \text { for } e \subset E, \text { such as } e=\{k, k n\} . \\
\text { For } p & =0, \text { finally } \\
\left\langle w^{N} ; n\right\rangle & =1 \quad \text { for } n \text { at } N ; \\
\left\langle w^{N} ; n\right\rangle=\frac{1}{2} & \text { for all } n \text { mid-points of edges with one } \\
& \text { extremity at } N, \text { such as } k n, k l \text { or } k m .
\end{aligned}
$$

Remark 5.3. The strategy adopted to compute the coefficients $\pi_{s}^{S}, S \in \mathcal{S}_{m}^{p}$ and $s \in \mathcal{S}_{\tilde{m}}^{p}$, can be used when dealing with quadratic, cubic, etc., differential forms, provided that the integration rule is modified accordingly. Therefore, the main problem with Whitney elements of order $r>1$ (see, e.g., [9]) is the definition on a $p$-simplex, $p=2,3$, of an integration rule which is exact for all polynomials of degree $r$ (on 1-simplices we can use Gaussian quadratures). This problem is far from being trivial and is linked to another one, namely, the location in a $p$-simplex of the degrees of 
freedom associated with Whitney elements of order $r>1$. Both problems will be addressed in future work.

As we have stressed, computing the coefficients of the chain-map $\pi$ is a metricindependent process. Implementation, however, may have to be done in a code conceived in terms of proxy vector fields, with an underlying metric, instead of differential forms. Hence we have the following description of the procedure, where $|t|$ denotes the volume of tetrahedron $t,|f|$ the area of face $f$, and $|e|$ the length of edge $e$. We use $\mathbf{x}_{e}, \mathbf{x}_{f}, \mathbf{x}_{t}$ to denote the barycenters of edge $e$, face $f$, and tetrahedron $t$, respectively. Points $\mathbf{x}_{k}, \mathbf{x}_{\ell}, \mathbf{x}_{m}, \mathbf{x}_{n}$ are the vertices of $t$ or $T$. Moreover, $\mathbf{t}_{e}$ denotes the unit vector along the mesh side $e$, and $\mathbf{n}_{f}$ stands for the unit vector normal to the mesh face $f$. For completeness, we throw in the computation of the other chain map, $\chi$. Thanks to Lemmas 5.1 and 5.2, the following algorithm, though relying on metric elements such as dot product, etc., does implement in the nested case (up to floating-point errors, and barring clerical mistakes of ours ...) the metric-free computation of the prolongation/restriction operator we have detailed.

Loop over $S$, the $p$-simplices of $m$

Loop over $s \subset S$, with $s$ the $p$-simplices of $\tilde{m}$

Computation of $\pi_{s}^{S}$

$$
\begin{array}{ccc}
p=0, & \pi_{n}^{N}=w^{N}\left(\mathbf{x}_{n}\right), & S=N, s=n ; \\
p=1, & \pi_{e}^{E}=|e|\left(w^{E}\left(\mathbf{x}_{e}\right) \cdot \mathbf{t}_{e}\right), & S=E, s=e ; \\
p=2, & \pi_{f}^{F}=|f|\left(w^{F}\left(\mathbf{x}_{f}\right) \cdot \mathbf{n}_{f}\right), & S=F, s=f ; \\
p=3, & \pi_{t}^{T}=|t|, & S=T, s=t .
\end{array}
$$

Computation of $\chi_{S}^{s}$

$$
\begin{array}{lll}
\begin{array}{l}
p=0, \\
p=1,
\end{array} & \chi_{N}^{n}=1 & S=N, s=n, n \equiv N ; \\
p=2, & \chi_{E}^{e}=1(-1) & S=E, s=e, \mathbf{t}_{e} \cdot \mathbf{t}_{E}>0(<0), \\
p=3, & \chi_{F}^{f}=1(-1) & S=F, s=f, \mathbf{n}_{f} \cdot \mathbf{n}_{F}>0(<0), \\
& \chi_{T}^{t}=1(-1) & S=T, s=t \text { and }
\end{array}
$$

end loop over $s$

end loop over $S$.

\section{REFERENCES}

[1] M. A. Armstrong, Basic Topology, Springer-Verlag, New York, 1983.

[2] M. Berger and B. Gostiaux, Géométrie différentielle: variétés, courbes et surfaces, Presses Universitaires de France, Paris, 1987.

[3] P. B. Bochev, C. J. Garasi, J. J. Hu, A. C. Robinson, and R. S. Tuminaro, An improved algebraic multigrid method for solving Maxwell's equations, SIAM J. Sci. Comp., 25 (2003), pp. 623-642.

[4] A. Bossavit, Computational Electromagnetism: Variational Formulations, Complementarity, Edge Elements, Academic Press, New York, 1998.

[5] A. Bossavit, Generating Whitney forms of polynomial degree one and higher, IEEE Trans. Magn., 38 (2002), pp. 341-344.

[6] M. Clemens, S. Feigh, And T. Weiland, Geometric multigrid algorithms using the conformal finite integration technique, IEEE Trans. Magn., 40 (2004), pp. 1065-1068.

[7] J. HARrison, Stokes' theorem for nonsmooth chains, Bull. Amer. Math. Soc. (N.S.), 29 (1993), pp. 235-242.

[8] R. Hiptmain, Multigrid method for Maxwell's equations, SIAM J. Numer. Anal., 36 (1998), pp. 204-225. 
[9] J. C. NÉDÉLEC, Mixed finite elements in $\mathbb{R}^{3}$, Numer. Math., 35 (1980), pp. 315-341.

[10] J. C. NÉDÉLEC, A new family of mixed finite elements in $\mathbb{R}^{3}$, Numer. Math., 35 (1986), pp. 5781.

[11] G. Nicolas, F. Arnoux-Guisse, and O. Bonnin, Adaptive meshing for $3 D$ finite element software, in 9th International Conference in Finite Elements in Fluids, Venezia, 1995.

[12] F. Rapetti, F. Dubois, and A. Bossavit, Integer matrix factorization for mesh defects detection, C. R. Acad. Sci. Paris Sér. I math., 334 (2002), pp. 717-720.

[13] S. M. Rao, D. R. Wilton, and A. W. Glisson, Electromagnetic scattering by surfaces of arbitrary shape, IEEE Trans. Antennas and Propagation, 30 (1982), pp. 409-418.

[14] S. Reitzinger AND J. SchöBerl, An algebraic multigrid method for finite element discretizations with edge elements, Numer. Linear Algebra Appl., 9 (2002), pp. 223-238.

[15] S. SuURINIEMI, Homological Computations in Electromagnetic Modeling, Ph.D. thesis, Tampere University of Technology, Tampere, Finland, 2004.

[16] B. Smith, P. Bjørstad, And W. Gropp, Domain Decomposition, Cambridge University Press, New York, 1996.

[17] J. Stillwell, Classical Topology and Combinatorial Group Theory, Grad. Texts in Math. 72, Springer-Verlag, 1993.

[18] H. Whitney, Geometric Integration Theory, Princeton University Press, 1957.

[19] B. I. Wohlmuth, Discretization Methods and Iterative Solvers Based on Domain Decomposition, Lect. Notes Comput. Sci. Eng. 17, Springer, Heidelberg, 2001.

[20] K. Yosida, Functional Analysis, 6th ed., Springer, 1980. 